\title{
VANISHING IN STABLE MOTIVIC HOMOTOPY SHEAVES
}

\author{
KYLE ORMSBY ${ }^{1}$, OLIVER RÖNDIGS $^{2}$ and PAUL ARNE $Ø S T V Æ R^{3}$ \\ ${ }^{1}$ Reed College, USA; \\ email: ormsbyk@ reed.edu \\ ${ }^{2}$ Universität Osnabrück, Germany; \\ email: oroendig@uni-osnabrueck.de \\ ${ }^{3}$ University of Oslo, Norway; \\ email:paularne@math.uio.no
}

Received 24 April 2017; accepted 24 January 2018

\begin{abstract}
We determine systematic vanishing regions for the bigraded homotopy sheaves of the motivic sphere spectrum over a field of characteristic different from two.

2010 Mathematics Subject Classification: 14F42 (primary)
\end{abstract}

\section{Introduction}

Stable motivic homotopy theory is a nonabelian generalization of the algebrogeometric theory of motives. It is a natural arena in which to study the motivic cohomology, $K$-theory, and algebraic cobordism of smooth schemes [32], and its invention was crucial to the resolution of the Milnor and Bloch-Kato conjectures $[34,35]$.

The most fundamental objects in the stable motivic homotopy category $\mathrm{SH}^{\mathbb{A}^{1}}(F)$ (over a field $F$ ) are the $\mathbb{P}^{1}$-suspension spectra $\Sigma_{\mathbb{P}^{1}}^{\infty} U_{+}$for $U$, a smooth $F$ scheme. Distinguished among these is the sphere spectrum $\mathbb{1}:=\Sigma_{\mathbb{P} 1}^{\infty} \operatorname{Spec}(F)_{+}$. We denote this object by $\mathbb{1}$ because it is the unit for the symmetric monoidal product on $\mathrm{SH}^{\mathbb{A}^{1}}(F)$ given by the smash product.

Equivalence between $\mathbb{P}^{1}$-spectra is detected by the bigraded homotopy sheaves, $\underline{\pi}_{m+n \alpha} X$ for $m, n \in \mathbb{Z}$, which are defined as the Nisnevich sheafification of the

(c) The Author(s) 2018. This is an Open Access article, distributed under the terms of the Creative Commons Attribution licence (http://creativecommons.org/licenses/by/4.0/), which permits unrestricted re-use, distribution, and reproduction in any medium, provided the original work is properly cited. 
assignment

$$
U \in \operatorname{Sm} / \operatorname{Spec}(F) \longmapsto\left[\Sigma^{m+n \alpha} U_{+}, X\right]_{\mathrm{SH}^{\mathbb{A}^{1}}(F)} \cdot
$$

Here $[,]_{\mathrm{SH}^{\mathbb{A}^{1}}(F)}$ denotes the hom-set in $\operatorname{SH}^{\mathbb{A}^{1}}(F)$ and $\Sigma^{m+n \alpha}$ denotes smashing with $\left(S^{1}\right)^{\wedge m} \wedge\left(\mathbb{A}^{1} \backslash 0\right)^{\wedge n}$. Since every motivic spectrum is a $\mathbb{1}$-module, the bigraded sheaf

$$
\underline{\pi}_{\star} \mathbb{1}=\bigoplus_{m, n \in \mathbb{Z}} \underline{\pi}_{m+n \alpha} \mathbb{1}
$$

plays a fundamental role in stable motivic homotopy theory, analogous to the stable homotopy groups of spheres in topology. We will refer to $\underline{\pi}_{m+n \alpha} \mathbb{1}$ as the

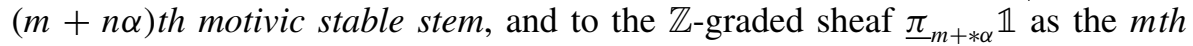
Milnor-Witt stem.

The motivic stable stems (and their global sections, $\pi_{m+n \alpha} \mathbb{1}:=\underline{\pi}_{m+n \alpha} \mathbb{1}(F)$ ) have been objects of intense study since Morel's analysis of the Oth motivic stable stem in [17]. That paper launched his program [18] to identify the 0th Milnor-Witt stem with $\underline{K}_{-*}^{M W}$, the Milnor-Witt $K$-theory sheaf, explaining the nomenclature. In further work [19], Morel shows that $\mathbb{1}$ is connective, meaning that $m$ th MilnorWitt stems are 0 for $m<0$.

Beyond Morel's theorems, little is known about Milnor-Witt stems over a general field. Röndigs-Spitzweck-Østvær [26] determine the first Milnor-Witt stem as an extension of $\underline{K}_{*}^{M} / 24$ and a certain sheaf related to Hermitian $K$ theory; this vastly generalizes the work of Ormsby-Østvær [23] for fields of cohomological dimension less than three. All other computations are limited to specific fields, and are generally only known on global sections (and frequently after completion at 2). Indeed, Hu-Kriz-Ormsby [14] and Dugger-Isaksen [4] make computations over $\mathbb{C}$ via the Adams-Novikov and Adams spectral sequences, Ormsby [22] makes computations over $p$-adic fields, Heller-Ormsby $[9,10]$ and Dugger-Isaksen [5, 6] make computations over $\mathbb{R}$, and Wilson-Østvær [37] over finite fields. All these computations hold only in specific (often finite) ranges.

In this paper, we exploit the methods of [26] to find conditions under which the $m$ th Milnor-Witt stem is bounded above; see Theorems 1.4 and 1.6. Our methods apply to a general field $F$ of characteristic different from 2 , and they result in sheaf level theorems (after inverting $\operatorname{char}(F)$ if $\operatorname{char}(F)$ is odd).

Our vanishing theorems have important implications for the nonzero homotopy sheaves of $\mathbb{1}$ via Morel's contraction construction [20]. Given a Nisnevich sheaf of abelian groups $\mathscr{F}$ on $\mathrm{Sm} / F$, the contraction $\omega \mathscr{F}$ of $\mathscr{F}$ takes $U$ to the kernel of $\mathscr{F}\left(U \times\left(\mathbb{A}^{1} \backslash 0\right)\right) \rightarrow \mathscr{F}(U)$. (Here the map is induced by the canonical 
section $1: \operatorname{Spec} F \rightarrow \mathbb{A}^{1} \backslash 0$.) For any motivic spectrum $E$, we have $\omega \underline{\pi}_{m+n \alpha} E \cong$ $\underline{\pi}_{m+(n+1) \alpha} E$. In particular, if $\underline{\pi}_{m+n \alpha} E=0$, then, for $k \geqslant 1$, the $k$-fold contraction of $\underline{\pi}_{m+(n-k) \alpha} E$ is 0 . Future computations should be able to exploit vanishing of $\underline{\pi}_{m+n \alpha} \mathbb{1}$ to constrain the structure of $\underline{\pi}_{m+\ell \alpha} \mathbb{1}$ for $\ell<n$.

We now state our results precisely, giving some indication of our methods along the way. Fix a field $F$ and let $q$ denote its exponential characteristic. We begin by studying the $\eta$-complete sphere spectrum via Voevodsky's slice spectral sequence [33] using the results in [26], and then 'uncomplete' our results via a sequence of fracture squares.

Let $\eta \in \underline{\pi}_{\alpha} \mathbb{1}$ (Spec $F$ ) denote the motivic Hopf map induced by the projection $\mathbb{A}^{2} \backslash 0 \rightarrow \mathbb{P}^{1}$. The $\eta$-complete sphere is the motivic spectrum $\hat{\mathbb{1}}=\operatorname{holim}_{n} \mathbb{1} / \eta^{n}$. Theorem 1.1 will be shown in Section 3 .

THEOREM 1.1. Over a field $F$ with exponential characteristic $q \neq 2$,

$$
\underline{\pi}_{m+n \alpha} \hat{\mathbb{1}}[1 / q]=0
$$

whenever

$» m<0$, or

$» m>0, m \equiv 1$ or $2(\bmod 4)$, and $2 n>\max \{3 m+5,4 m\}$.

Using the same techniques that prove Theorem 1.1, we can prove a stronger vanishing result for $\hat{\mathbb{1}}_{(p)}$, the $\eta$-completion of the $p$-local sphere spectrum. (The notation $\widehat{\mathbb{1}_{(p)}}$ might be more appropriate, but we find it unwieldy.) Theorem 1.2 will be shown in Section 3.

THEOREM 1.2. Let $F$ be a field and let $p$ be an odd prime different from the characteristic of $F$. Then

$$
\underline{\pi}_{m+n \alpha} \hat{\mathbb{1}}_{(p)}=0
$$

whenever

$» m<0$, or

» $m \geqslant 0$ and $(p-2) n>(p-1) m$.

While the $\eta$-complete sphere is an interesting object in its own right, one would like to know if there are vanishing regions in $\underline{\pi}_{\star} \mathbb{1}$ as well. When the cohomological dimension $\mathrm{cd} F<\infty$, this is known by the following theorem, essentially due to Levine [15]. 
THEOREM 1.3. Suppose $F$ is perfect and $\mathrm{cd} F<\infty$. Then $\mathbb{1} \simeq \hat{\mathbb{1}}$.

Proof. In [15], Levine proves that the slice spectral sequence for $\mathbb{1}$ converges to $\pi_{\star} \mathbb{1}$ when cd $F<\infty$. Meanwhile, [26, Theorem 3.50] identifies the target with $\underline{\pi}_{\star} \hat{\mathbb{1}}$, so the completion map $\mathbb{1} \rightarrow \hat{\mathbb{1}}$ induces an isomorphism $\underline{\pi}_{\star} \mathbb{1} \cong \underline{\pi}_{\star} \hat{\mathbb{1}}$ and thus $\mathbb{1} \simeq \hat{\mathbb{1}}$.

From this, we deduce Theorem 1.4 in Section 4.1. By a 'nonreal field' we mean a field that is not formally real.

THEOREM 1.4. Suppose $F$ is nonreal with exponential characteristic $q \neq 2$. If $q>2$, further suppose that $F$ is perfect and of finite cohomological dimension. Then $\underline{\pi}_{m+n \alpha} \mathbb{1}[1 / q]$ vanishes in the range given in Theorem 1.1. If $p \neq q$ is an odd prime, then $\underline{\pi}_{m+n \alpha} \mathbb{1}_{(p)}$ vanishes in the range given in Theorem 1.2.

The positive characteristic statement is a direct consequence of Theorem 1.3, but the characteristic 0 nonreal case does not exclude the possibility of infinite cohomological dimension. We handle this via standard base change methods, which we explain in Section 4.1.

REMARK 1.5. Note that Theorem 1.4 covers all nonreal fields of characteristic 0 , and all odd characteristic (necessarily nonreal) fields that are perfect with finite cohomological dimension. We cover the case of formally real (necessarily characteristic 0) fields in Theorem 1.6.

When $F$ is formally real, vanishing in $\underline{\pi}_{\star} \mathbb{1}$ is more interesting. We first observe that the $\eta$-primary fracture square

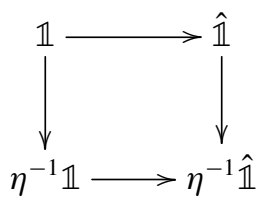

reduces the problem to that of vanishing regions for $\pi_{\star} \eta^{-1} \mathbb{1}$. We solve this problem using Bachmann's theorem on $\underline{\pi}_{\star} \mathbb{1}[1 / 2,1 / \eta]$ and the Hu-Kriz-Ormsby comparison of the 2 - and $(2, \eta)$-complete spheres when $F$ has finite virtual 2cohomological dimension. (Recall that $\operatorname{vcd}_{2}(F):=\operatorname{cd}_{2}\left(F(\sqrt{-1})\right.$ ), where $\operatorname{cd}_{2}$ denotes 2-primary étale cohomological dimension.) In order to state our results, let $\pi_{m}^{\text {top }} \mathbb{1}$ denote the $m$ th homotopy group of the topological sphere spectrum. Theorem 1.6 is shown in Section 4.3. 
THEOREM 1.6. Suppose $F$ is formally real. If $m<0, \underline{\pi}_{\star} \mathbb{1}=\underline{\pi}_{\star} \mathbb{1}_{(p)}=0$ by Morel's connectivity theorem. Suppose $m>0$. Then $\underline{\pi}_{m+n \alpha} \mathbb{1}=0$ whenever $\underline{\pi}_{m+n \alpha} \hat{\mathbb{1}}=0$ (see Theorem 1.1) and $\pi_{m}^{\mathrm{top}} \mathbb{1}[1 / 2]=0$. If $p$ is an odd prime, then $\underline{\pi}_{m+n \alpha} \mathbb{1}_{(p)}=0$ whenever $\underline{\pi}_{m+n \alpha} \hat{\mathbb{1}}_{(p)}=0$ (see Theorem 1.2$)$ and $\pi_{m}^{\text {top }} \mathbb{1}_{(p)}=0$.

Of course, determining when $\pi_{m}^{\text {top }} \mathbb{1}[1 / 2]$ or $\pi_{m}^{\text {top }} \mathbb{1}_{(p)}$ is 0 is no easy task. Nonetheless, we view these conditions as 'reductions to topology', which effectively transfers the problem from motivic to classical homotopy theory. Given the intractability of these topological vanishing problems, this is the best type of result we can hope for.

EXAMPLE 1.7. Suppose $F$ is formally real. Toda's calculations say that $\pi_{18}^{\text {top }} \mathbb{1}=$ $\mathbb{Z} / 8 \oplus \mathbb{Z} / 2[31$, p. 188$]$. Since $18 \equiv 2(\bmod 4)$ and $2 \cdot 37>4 \cdot 18$, Theorem 1.6 implies that $\underline{\pi}_{18+37 \alpha} \mathbb{1}=0$. Ravenel's calculations imply that $\pi_{61}^{\text {top }} \mathbb{1}[1 / 2]=0$ [24, Theorem 1.1.13, A3.4, A3.5, Theorem 4.4.20]. Since $61 \equiv 1(\bmod 4)$ and $2 \cdot 123>4 \cdot 61$, one obtains $\underline{\pi}_{61+123 \alpha} \mathbb{1}=0$.

REMARK 1.8. It is also possible to produce a relative version of Theorem 1.6, which specifies a range and the manner in which $\pi_{m+n \alpha} \mathbb{1}$ is 'topological' in the sense of being computed in the homotopy category of sheaves of spectra on the Harrison space $X_{F}$ of orderings of $F$. See Remark 5.7 for a precise statement.

CONVENTION 1.9. Henceforth, we always invert the exponential characteristic $q$ of the base field $F$, but we omit this from our notation. Note that when $F$ is formally real, $q=1$, so our theorems for formally real fields are genuinely integral.

REMARK 1.10. After completing the first draft of this paper, Bogdan Gheorghe and Dan Isaksen pointed out that their paper [7] contains a precedent for this style of vanishing theorem. They use the motivic Adams-Novikov spectral sequence to deduce an ' $\eta$-local' region in the global sections of the bigraded motivic homotopy sheaves of the 2-complete sphere spectrum over $\mathbb{C}$. The computation of $\underline{\pi}_{\star} \eta^{-1} \mathbb{1}(\operatorname{Spec} \mathbb{C})$ in $[1]$ then implies that $\underline{\pi}_{m+n \alpha} \hat{\mathbb{1}}(\operatorname{Spec} \mathbb{C})=0$ for $m \equiv 1$ or 2 $(\bmod 4)$ and $2 n>3 m+5$.

Further note that since $\underline{\pi}_{\star} \eta^{-1} \mathbb{1}(\operatorname{Spec} \mathbb{C}) \cong \mathbb{Z} / 2$ for $m \geqslant 0, m \equiv 0$ or 3 $(\bmod 4)$, our conditions on the congruence class of $m(\bmod 4)$ are in fact necessary. 
Outline. In Section 2, we collect some necessary facts about the slice and (Adams-)Novikov spectral sequences. In Section 3, we use the slice spectral sequence to prove Theorems 1.1 and 1.2. Section 4 is split into three subsections. In Section 4.1, we review some base change theorems and use them to prove Theorem 1.4. In Section 4.2, we review Bachmann's theorem on $\operatorname{SH}^{\mathbb{A}^{1}}(F)[1 / 2$, $1 / \eta]$. In Section 4.3, we use fracture square methods to prove Theorem 1.6. Finally, in Section 5, we pose several open questions and prove Theorem 5.5, which compares the motivic stable stems and $\eta$-inverted motivic stable stems in a range.

\section{Preliminaries}

In this section, we gather known facts about the slice and Novikov spectral sequences that we will need for our arguments.

We use the slice spectral sequence to prove Theorems 1.1 and 1.2. See [33] for its construction and [26] for a contemporary take on its properties. The slice spectral sequence for the sphere takes the form

$$
E_{1}^{m, n, t}=\underline{\pi}_{m+n \alpha} s_{t} \mathbb{1} \Longrightarrow \underline{\pi}_{m+n \alpha} \hat{\mathbb{1}},
$$

where $s_{t} \mathbb{1}$ is the $t$ th slice of the sphere spectrum [26, Theorem 3.50]. By loc. cit. applied to the admissible pair $\left(\operatorname{Spec} F, \mathbb{Z}_{(p)}\right)$, its $p$-local analogue takes the form

$$
E_{1}^{m, n, t}(p)=\underline{\pi}_{m+n \alpha} s_{t} \mathbb{1}_{(p)} \Longrightarrow \underline{\pi}_{m+n \alpha} \hat{\mathbb{1}}_{(p)}
$$

whenever $p \neq \operatorname{char} F$. In the case of Theorem 1.2, we prove that $E_{1}^{m, n, t}(p)$ vanishes in the stated range, implying that $\pi_{\star}{ }_{\star} \hat{\mathbb{1}}_{(p)}$ vanishes in the same range. In the case of Theorem 1.1, we must work a little harder and show that $E_{2}^{m, n, t}$ vanishes in appropriate regions.

Both proofs depend crucially on the form that the slices of $\mathbb{1}$ take. Surprisingly, these slices are governed by the $E_{2}$-page of the Novikov (that is, MU-Adams) spectral sequence from classical stable homotopy theory. Let

$$
E_{2}^{s, t}(\mathrm{MU})=\mathrm{Ext}_{\mathrm{MU}_{*} \mathrm{MU}}^{\mathrm{M}}\left(\mathrm{MU}_{*}, \mathrm{MU}_{*}\right)
$$

denote the cohomology of the MU Hopf algebroid, where $s$ denotes homological degree and $t$ the internal grading on $\mathrm{MU}_{*}$. (Note that $\mathrm{MU}_{*}$ is even-graded, so this group vanishes whenever $t$ is odd.) Furthermore, let $\mathbf{M}$ denote the motivic Eilenberg-MacLane functor, which takes in an abelian group $A$ and produces the spectrum $\mathbf{M} A$ representing motivic cohomology with coefficients in $A$. Using this notation, we get the following theorem due to Röndigs-Spitzweck-Østvær. 
THEOREM 2.1 [26, Theorem 2.12]. The th slice of the motivic sphere spectrum is

$$
s_{t} \mathbb{1}=\bigvee_{s \geqslant 0} \Sigma^{t-s+t \alpha} \mathbf{M} E_{2}^{s, 2 t}(\mathrm{MU})
$$

To further understand the $E_{1}$-page of the slice spectral sequence, we will need more information on two things: first, the homotopy sheaves of motivic EilenbergMacLane spectra, and second, structural properties of $E_{2}^{s, t}(\mathrm{MU})$.

LEMMA 2.2 [29, Corollary 3.2.1]. Suppose A is a finitely generated abelian group. Then

$$
\underline{\pi}_{m+n \alpha} \mathbf{M} A=0
$$

for $m<0$, or $m=0$ and $n>0$, or $m>0$ and $n>-1$.

Proof. We have

$$
\begin{aligned}
\left(\underline{\pi}_{m+n \alpha} \mathbf{M} A\right)(U) & =\left[S^{m+n \alpha} \wedge U_{+}, \mathbf{M} A\right] \\
& \cong\left[U_{+}, S^{-m-n \alpha} \wedge \mathbf{M} A\right] \\
& \cong H^{-m-n}(U ; A(-n)) .
\end{aligned}
$$

The stated vanishing range then follows from [29, Corollary 3.2.1].

While discussing motivic Eilenberg-MacLane spectra, we take a moment to note the following lemma, which we will need later in our arguments. Recall that $\tau \in \pi_{1-\alpha} \mathbf{M} \mathbb{F}_{2}$ is the element represented by -1 in the kernel of the squaring map on the units of a field $F$ of char $F \neq 2$.

LEMma 2.3. Suppose char $F \neq 2$. Then multiplication by $\tau$ is injective on $\underline{\pi}_{\star} \mathbf{M} \mathbb{F}_{2}$.

Proof. It suffices to prove that multiplication by $\tau$ is injective on the stalks of $\pi_{\star} \mathbf{M F}_{2}$. These stalks are given by evaluating $\underline{\pi}_{\star} \mathbf{M} \mathbb{F}_{2}$ at the henselization $\mathcal{O}_{X, x}^{h}$ of the local ring at a point $x$ on a smooth $F$-scheme $X$. By Suslin-Voevodsky rigidity [30, Theorem 4.4] and [11], the map from $\mathcal{O}_{X, x}^{h}$ to its residue field induces an isomorphism on motivic cohomology with $\mathbb{F}_{2}$-coefficients. Hence it suffices to prove that multiplication by $\tau$ is injective on the motivic cohomology with $\mathbb{F}_{2}$-coefficients of any field extension $E$ of $F$. By the solution of the Milnor conjecture, $\underline{\pi}_{\star} \mathbf{M} \mathbb{F}_{2}(\operatorname{Spec} E) \cong K_{*}^{M}(E) / 2[\tau]$, so injectivity of $\tau$ is obvious.

We now turn to the structure of $E_{2}^{s, t}(\mathrm{MU})$. This has been an object of intense study since the 1970s, and the results we need are easily culled from the literature. 
We first consider various finiteness properties, and how to build up $E_{2}^{s, t}$ (MU) from $p$-local information.

LEMMA 2.4.

(a) Unless $(s, t)=(0,0)$, the group $E_{2}^{s, t}(\mathrm{MU})$ is finite; furthermore, $E_{2}^{0,0}(M U)=\mathbb{Z}$

(b) Let $\mathscr{P}$ denote the set of rational primes and for $p \in \mathscr{P}$ let $\mathrm{BP}(p)$ denote the p-local Brown-Peterson spectrum. Let $E_{2}^{*, *}(\mathrm{BP}(p))$ denote the cohomology of the $\mathrm{BP}(p)$ Hopf algebroid. Then

$$
E_{2}^{>0, *}(\mathrm{MU}) \cong \bigoplus_{p \in \mathscr{P}} E_{2}^{>0, *}(\mathrm{BP}(p))
$$

(c) There is a vanishing line so that $E_{2}^{s, t}(\mathrm{BP}(p))=0$ when $t<2 s(p-1)$.

Proof. These are all standard results going back to Novikov and Zahler. For (a), see [21, Proposition 2.1]. For (b), see [38, p. 482]. For (c), see [21, Corollary 3.1].

REMARK 2.5. Readers expert in the Adams-Novikov spectral sequence will notice that we did not include the sparsity theorem $E_{2}^{s, t}(\mathrm{BP}(p))=0$ whenever $2 p-2 \nmid t$. Combined with Theorem 2.1, sparsity certainly gives interesting information about the suspension bigrading of $p$-local slice summands. But because of the fourth quadrant cone worth of nonzero homotopy sheaves associated with an Eilenberg-MacLane spectrum (Lemma 2.2), we do not get analogous sparsity results on $\underline{\pi}_{\star} \hat{\mathbb{1}}_{(p)}$, at least when $\operatorname{cd} F=\infty$. If $\operatorname{cd} F$ is finite and sufficiently small relative to $p$, then one can deduce a sort of sparsity result for $\underline{\pi}_{\star} \mathbb{1}$, but we do not pursue the specifics here.

Finally, we will need to leverage the Andrews-Miller analysis of the $\alpha_{1}$-inverted 2-local Adams-Novikov spectral sequence [1]. Recall that $\alpha_{1}$ is the generator of $E_{2}^{1,2}(\mathrm{BP}(2)) \cong E_{2}^{1,2}(\mathrm{MU})$.

LEMMA 2.6. There is an isomorphism

$$
\alpha_{1}^{-1} E_{2}^{*, *}(\mathrm{MU}) \cong \mathbb{F}_{2}\left[\alpha_{1}^{ \pm 1}, \alpha_{3}, \alpha_{4}\right] /\left(\alpha_{4}^{2}\right)
$$

where $\left|\alpha_{3}\right|=(1,6)$ and $\left|\alpha_{4}\right|=(1,8)$. Moreover, the localization map

$$
E_{2}^{s, t}(\mathrm{MU}) \rightarrow \alpha_{1}^{-1} E_{2}^{s, t}(\mathrm{MU})
$$

is an isomorphism whenever $t<6 s-10$ and $t<4 s$. 
Proof. In [1, Corollary 6.2.3], Andrews and Miller prove that

$$
\alpha_{1}^{-1} E_{2}^{*, *}(\mathrm{BP}(2)) \cong \mathbb{F}_{2}\left[\alpha_{1}^{ \pm 1}, \alpha_{3}, \alpha_{4}\right] /\left(\alpha_{4}^{2}\right) .
$$

Since $2 \alpha_{1}=0$, the $E_{2}^{*, *}(\mathrm{MU})$ version of this isomorphism follows from Lemma 2.4(b).

For the second part of the lemma, note that by [1, Proposition 5.1],

$$
E_{2}^{s, t}(\mathrm{BP}(2)) \rightarrow \alpha_{1}^{-1} E_{2}^{s, t}(\mathrm{BP}(2))
$$

is an isomorphism when $t<6 s-10$. For $p>2$, Lemma 2.4(c) implies that $E_{2}^{s, t}(\mathrm{BP}(p))=0$ for $t<2 s(p-1) \leqslant 4 s$. Hence when $t<\min \{6 s-10,4 s\}$, we are guaranteed to only have 2-primary groups in the Andrews-Miller isomorphism range.

\section{Vanishing for the $\eta$-complete sphere}

This section consists of the proofs of Theorems 1.1 and 1.2.

Proof of Theorem 1.1. Vanishing for $m<0$ follows from the vanishing range in the $E_{1}$-page of the slice spectral sequence, which in turn relies on Morel's connectivity theorem [19]. We turn to the second condition, namely vanishing of $\underline{\pi}_{m+n \alpha} \hat{\mathbb{1}}$ when $m>0, m \equiv 1$ or $2(\bmod 4)$, and $2 n>\max \{3 m+5,4 m\}$. Recall that the slice spectral sequence

$$
E_{1}^{m, n, t}=\underline{\pi}_{m+n \alpha} s_{t} \mathbb{1} \Longrightarrow \underline{\pi}_{m+n \alpha} \hat{\mathbb{1}}
$$

converges to the homotopy sheaves of $\hat{\mathbb{1}}$. By Theorem 2.1, we may rewrite the $E_{1}$-page as

$$
E_{1}^{m, n, t}=\bigoplus_{s \geqslant 0} \underline{\pi}_{m+n \alpha} \Sigma^{t-s+t \alpha} \mathbf{M} E_{2}^{s, 2 t}(\mathrm{MU})
$$

Let $T$ denote the linear transformation of the $(s, t)$-plane to the $(m+n \alpha)$-plane given by the matrix $\left(\begin{array}{cc}-1 & 1 / 2 \\ 0 & 1 / 2\end{array}\right)$ (where both planes are given their standard bases). We call $T$ the Novikov-to-slice grading shift since Novikov $E_{2}$-terms in degree $(s, t)$ correspond to slice summands, which are Eilenberg-MacLane spectra shifted by $T(s, t)$. We say that a bigrading $m+n \alpha$ contains a slice summand if there exist integers $s, t$ such that $t-s+t \alpha=m+n \alpha$ and $E_{2}^{s, 2 t}(\mathrm{MU}) \neq 0$. By Theorem 2.1, $m+n \alpha$ contains a slice summand if and only if $E_{2}^{n-m, 2 n}(\mathrm{MU}) \neq 0$. We see then that under the Novikov-to-slice grading shift $T$, vanishing regions in $E_{2}^{s, t}(\mathrm{MU})$ are mapped to bigradings that do not contain a slice summand. 




Figure 1. This diagram represents structural features of $E_{2}^{s, t}(\mathrm{MU})$ and $E_{2}^{s, t}(\mathrm{BP}(p))$ for various primes $p$. Note that we have drawn the $E_{2}$-page in the tradition Adams grading with $t-s$ on the horizontal axis and $s$ on the vertical axis. The lines labeled by a prime $p$ correspond to $p$-local vanishing lines, so that $E_{2}^{s, t}(\mathrm{BP}(p))=0$ above these lines. The piecewise linear curve corresponds to the Andrews-Miller range of Lemma 2.6.

Similarly, other structural properties are preserved by $T$ as long as statements are translated into the language of slice summands (rather than groups or sheaves); see Figures 1 and 2. For instance, let $A$ denote the region in the $(s, t)$-plane specified in Lemma 2.6 in which the map $E_{2}^{s, t}(\mathrm{MU}) \rightarrow \alpha_{1}^{-1} E_{2}^{s, t}(\mathrm{MU})$ is an isomorphism; that is,

$$
A=\{(s, t) \mid t<\min \{6 s-10,4 s\}\} .
$$

Then within the region

$$
T(A)=\{m+n \alpha \mid 2 n>\max \{3 m+5,4 m\}\},
$$

we know that every nontrivial slice summand is a suspension of $\mathbf{M} \mathbb{F}_{2}$ indexed by a monomial of the form $\alpha_{1}^{i} \alpha_{3}^{j} \alpha_{4}^{\varepsilon}$, where $i$ and $j$ are sufficiently large integers and $\varepsilon=0$ or 1 . The suspension bigrading for an $\mathbf{M} \mathbb{F}_{2}$ indexed by $\alpha_{1}^{i} \alpha_{3}^{j} \alpha_{4}^{\varepsilon}$ is $(2 j+3 \varepsilon)+(i+3 j+4 \varepsilon) \alpha$.

We now show that in the slice spectral sequence we have

$$
E_{2}^{m, n, t}=0
$$

when $m+n \alpha \in T(A)$ and $m \equiv 1$ or $2(\bmod 4)$. By [26, Lemma 4.2], there are $d_{1}$ differentials in the slice spectral sequence, which restrict to

$$
\tau \text { pr : } \Sigma^{4 q+1+(4 q+2) \alpha} \mathbf{M} \mathbb{Z} / a_{2 q} \mathbb{Z} \rightarrow \Sigma^{4 q+(4 q+3) \alpha} \mathbf{M} \mathbb{F}_{2}
$$




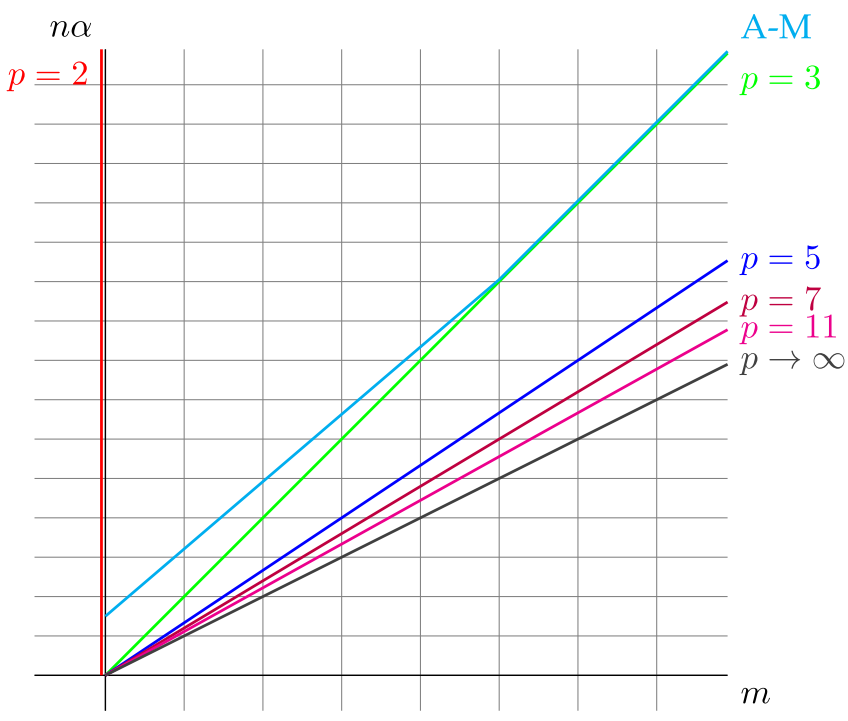

Figure 2. This diagram represents structural features of the $E_{1^{-}}$and $E_{2}$-pages of the slice spectral sequence for the motivic sphere spectrum. The $m$ - and $n \alpha-$ axes are presented, with the slice grading $t$ suppressed. After the Novikov-to-slice grading shift, the $p$-local vanishing lines of Figure 1 become the vanishing lines of Theorem 1.2, and the Andrews-Miller vanishing curve gives the vanishing curve of Theorem 1.1 (for $m \equiv 1$ or $2(\bmod 4)$ ). Note that the vanishing range given by Theorem 1.1 is much better than the naive range given by the $p=2$ case of Theorem 1.2.

on $\bar{\alpha}_{4 q+2}$,

$$
\tau: \Sigma^{4 q+1+(4 q+2+j) \alpha} \mathbf{M} \mathbb{F}_{2} \rightarrow \Sigma^{4 q+(4 q+3+j) \alpha} \mathbf{M} \mathbb{F}_{2}
$$

on $\alpha_{1}^{j} \alpha_{4 q+2}$ for $j \geqslant 1$, and

$$
\tau: \Sigma^{4 q-2+(4 q-1+j) \alpha} \mathbf{M} \mathbb{F}_{2} \rightarrow \Sigma^{4 q-3+(4 q+j) \alpha} \mathbf{M} \mathbb{F}_{2}
$$

on $\alpha_{1}^{j} \alpha_{4 q-1}$ for $j \geqslant 0$. Within $T(A)$, these differentials are multiplication by $\tau$ linking suspended $\mathbf{M} \mathbb{F}_{2}$ summands indexed by $\alpha_{1}^{i} \alpha_{3}^{2 j+1} \alpha_{4}^{\varepsilon}$ to suspended $\mathbf{M} \mathbb{F}_{2}$ summands indexed by $\alpha_{1}^{4+i} \alpha_{3}^{2 j} \alpha_{4}^{\varepsilon}$. By Lemma 2.3, multiplication by $\tau$ is injective on $\pi_{\star}{ }_{\star} M \mathbb{F}_{2}$. It follows that $E_{2}^{T(A), *}$ is concentrated in columns indexed by $m \equiv$ 0 or $3(\bmod 4)$.

This gives our desired vanishing result on the slice $E_{2}$-page, which in turn implies that $\underline{\pi}_{m+n \alpha} \hat{\mathbb{1}}=0$ when $m+n \alpha \in T(A)$ and $m \equiv 1$ or $2(\bmod 4)$, concluding our proof. 
Proof of Theorem 1.2. Consider the $p$-local slice spectral sequence

$$
E_{1}^{m, n, t}(p)=\underline{\pi}_{m+n \alpha} s_{t} \mathbb{1}_{(p)} \Longrightarrow \underline{\pi}_{m+n \alpha} \hat{\mathbb{1}}_{(p)} .
$$

A $p$-local version of Theorem 2.1 implies that

$$
s_{t} \mathbb{1}_{(p)}=\bigvee_{s \geqslant 0} \Sigma^{t-s+t \alpha} \mathbf{M} E_{2}^{s, 2 t}(\mathrm{BP}(p)) .
$$

By Lemma 2.4(c), $E_{2}^{s, t}(\mathrm{BP}(p))=0$ for $t<2 s(p-1)$. Under the Novikov-to-slice grading shift $T$ (see the proof of Theorem 1.1), this region becomes

$$
\{m+n \alpha \mid(p-2) n>(p-1) m\} .
$$

As such,

$$
s_{n} \mathbb{1}_{(p)} \simeq *
$$

for $(p-2) n>(p-1) m$. By Lemma 2.2, a nontrivial slice summand $\Sigma^{t-s+t \alpha} \mathbf{M} E_{2}^{s, 2 t}(\mathrm{BP}(p))$ can only contribute to $E_{1}^{m, n, t}(p)$ when $m-t+s \geqslant 0$ and $n-t \leqslant 0$. (Lemma 2.2 actually provides a more stringent vanishing condition, but this 'nonfourth quadrant' vanishing is all we need here.) It follows that $E_{1}^{m, n, t}(p)=0$ for $(p-2) n>(p-1) m$. We conclude that $\underline{\pi}_{m+n \alpha} \hat{\mathbb{1}}_{(p)}=0$ in this range as well.

\section{Vanishing for the integral and $p$-local spheres}

In this section, we study the problem of lifting vanishing results about $\underline{\pi}_{\star} \hat{\mathbb{1}}$ to $\pi_{\star} \mathbb{1}$. We first recall some base change theorems and use them to prove Theorem 1.4. We then recall Bachmann's theorem on $\operatorname{SH}^{\mathbb{A}^{1}}(F)[1 / 2,1 / \eta]$, and finally prove Theorem 1.6.

4.1. Base change. Recall that for any map of schemes $f: S \rightarrow T$ one has a pullback, that is, base change, functor $f^{*}: \mathrm{SH}^{\mathbb{A}^{1}}(T) \rightarrow \operatorname{SH}^{\mathbb{A}^{1}}(S)$. In this subsection, we use standard arguments with base change functors to expand the class of fields for which various vanishing results will hold. We write $\mathbb{1}_{F}$ for the sphere spectrum in $\mathrm{SH}^{\mathbb{A}^{1}}(F)$ or $\mathrm{SH}^{\mathbb{A}^{1}}(F)[1 / q]$. Note that if $f: \operatorname{Spec} E \rightarrow \operatorname{Spec} F$ is an extension of fields, then $f^{*} \mathbb{1}_{F}=\mathbb{1}_{E}$.

The functor $f^{*}$ always admits a right adjoint $f_{*}$. If $f$ is smooth, it also admits a left adjoint $f_{\sharp}$ (given by composition of the structure map to $S$ with $f$ ). (See [12, Appendix A] for a brief review.)

Below we will use the fact that when $F$ is perfect, $\pi_{m+n \alpha} X$ is a strictly $\mathbb{A}^{1}$ invariant sheaf in the sense of [20]. (See [12, Section 1.2] for a brief review.) 
We call an extension of fields $E / F$ essentially smooth if Spec $E$ is a cofiltered limit of smooth $F$-schemes. Note that if the transcendence degree of $E / F$ is finite, then $E / F$ is essentially smooth.

LEMMA 4.1. Let $F$ be a perfect field and suppose $E / F$ is an essentially smooth field extension. Then there is an isomorphism $\underline{\pi}_{\star} \mathbb{1}_{F}(\operatorname{Spec} E) \cong \underline{\pi}_{\star} \mathbb{1}_{E}(\operatorname{Spec} E)$.

Proof. Write Spec $E$ as a cofiltered $\operatorname{limit}_{\lim _{\beta}} X_{\beta}$ of smooth $F$-schemes $X_{\beta}$. Fix $m, n \in \mathbb{Z}$. We have

$$
\begin{aligned}
\underline{\pi}_{m+n \alpha} \mathbb{1}_{F}(\operatorname{Spec} E) & =\operatorname{colim}_{\beta} \underline{\pi}_{m+n \alpha} \mathbb{1}_{F}\left(X_{\beta}\right) \\
& =\operatorname{colim}_{\beta}\left[\Sigma^{m+n \alpha} X_{\beta+}, \mathbb{1}_{F}\right] \\
& =\operatorname{colim}_{\beta}\left[f_{\beta \sharp} f_{\beta}^{*} \Sigma^{m+n \alpha} \mathbb{1}_{F}, \mathbb{1}_{F}\right] \\
& \cong \operatorname{colim}_{\beta}\left[f_{\beta}^{*} \Sigma^{m+n \alpha} \mathbb{1}_{F}, f_{\beta}^{*} \mathbb{1}_{F}\right] \\
& \cong\left[\Sigma^{m+n \alpha} \mathbb{1}_{E}, f^{*} \mathbb{1}_{F}\right] \\
& =\underline{\pi}_{m+n \alpha} \mathbb{1}_{E}(\operatorname{Spec} E),
\end{aligned}
$$

as desired.

In the following proposition, we write $\pi_{m+n \alpha} X:=\underline{\pi}_{m+n \alpha} X(\operatorname{Spec} F)$ for the $(m+n \alpha)$ th homotopy group (as opposed to sheaf) of $X \in \mathrm{SH}^{\mathbb{A}^{1}}(F)$.

Proposition 4.2. Suppose $F$ is a filtered colimit of fields $F=\operatorname{colim}_{\beta} F_{\beta}$ such that $\pi_{m+n \alpha} \mathbb{1}_{F_{\beta}}=0$ for all $\beta$. Then $\pi_{m+n \alpha} \mathbb{1}_{F}=0$.

Proof. This follows from [12, Lemma A.7(1)].

LeMmA 4.3. Fix $m, n \in \mathbb{Z}$ and suppose $\underline{\pi}_{m+n \alpha} \mathbb{1}_{k}=0$ for all nonreal characteristic 0 fields $k$ with $\mathrm{cd} k<\infty$. Then $\underline{\pi}_{m+n \alpha} \mathbb{1}_{F}=0$ for any nonreal characteristic 0 field $F$, regardless of cohomological dimension.

Proof. Since $\underline{\pi}_{m+n \alpha} \mathbb{1}_{F}$ is strictly $\mathbb{A}^{1}$-invariant [20, Remark 5.1.13], it suffices to check that $\underline{\pi}_{m+n \alpha} \mathbb{1}_{F}(\operatorname{Spec} L)=0$ for all finitely generated field extensions $L / F$. By Lemma 4.1 and [12, Lemma A.2], this is the same as showing $\pi_{m+n \alpha} \mathbb{1}_{L}=0$. Thus, by Proposition 4.2 and our hypothesis, it suffices to show that $L$ is a filtered colimit of nonreal fields with finite cohomological dimension.

Since $L$ is nonreal, there exist $a_{1}, \ldots, a_{n} \in L$ such that $-1=a_{1}^{2}+\cdots+a_{n}^{2}$. Let $L_{0}=\mathbb{Q}\left(a_{1}, \ldots, a_{n}\right)$. Then $L_{0}$ is nonreal and $\operatorname{cd} L_{0} \leqslant 2$ by [28, Section II.4.4, Proposition 13]. Letting $A$ range over finite subsets of $L \backslash L_{0}$ we see that 
$L=\operatorname{colim}_{A} L_{0}(A)$. By [28, Section II.4.1 Proposition 10' \& Section II.4.2 Proposition 11], we see that $\operatorname{cd} L_{0}(A)<\infty$, and it is clear that each $L_{0}(A)$ is nonreal, completing our proof.

We can now use Theorem 1.3 to prove Theorem 1.4.

Proof of Theorem 1.4. The positive characteristic statement is a direct consequence of Theorem 1.3. Suppose $F$ is nonreal with characteristic 0 . If $\operatorname{cd} F<\infty$, then Theorem 1.3 implies $\mathbb{1} \simeq \hat{\mathbb{1}}$, and Theorems 1.1 and 1.2 guarantee that $\pi_{\star} \mathbb{1}$ has the stated vanishing range. By Lemma 4.3 , this is enough to conclude that the same vanishing range holds when $\mathrm{cd} F=\infty$.

We conclude this section with a virtual cohomological dimension version of Lemma 4.3. We will use it in Section 4.3 to prove Theorem 1.6.

LEMMA 4.4. Fix $m, n \in \mathbb{Z}$ and suppose $\underline{\pi}_{m+n \alpha} \mathbb{1}_{k}=0$ for all formally real fields $k$ with $\operatorname{vcd}_{2} k<\infty$ and all nonreal characteristic 0 fields. Then $\underline{\pi}_{m+n \alpha} \mathbb{1}_{F}=0$ for any formally real field $F$, regardless of virtual 2-cohomological dimension.

Proof. Suppose $F$ is a formally real field and $L$ is a finitely generated extension of $F$. As in the proof of Lemma 4.3, it suffices to show $\pi_{m+n \alpha} \mathbb{1}_{L}=0$. If $L$ is nonreal, we are done by hypothesis, so we may assume $L$ is formally real. We aim to express $L$ as a filtered colimit of formally real fields with finite $\operatorname{vcd}_{2}$. Letting $A$ range over finite subsets of $L \backslash \mathbb{Q}$, we see that $L=\operatorname{colim}_{A} \mathbb{Q}(A)$. Each $\mathbb{Q}(A)$ is formally real (since it is a subfield of $L$ ) and since $\operatorname{vcd}_{2} \mathbb{Q}=2<\infty$, the same two propositions from [28] imply that $\operatorname{vcd}_{2} \mathbb{Q}(A)<\infty$ for each $A$. By Proposition 4.2, we are done.

4.2. Bachmann's theorem. Let $\rho$ denote the map $\mathbb{1} \rightarrow \Sigma^{\alpha} \mathbb{1}$ induced by taking the nonbasepoint of $S^{0}$ to $-1 \in \mathbb{A}^{1} \backslash 0$. In [3], Bachmann finds an alternate presentation of the $\rho$-inverted stable motivic homotopy category $\operatorname{SH}^{\mathbb{A}^{1}}(F)[1 / \rho]$ in terms of the real étale topology. We will not go into the details of the real étale topology, instead sending the reader to [27], especially its first chapter.

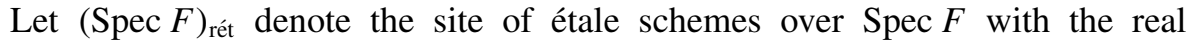
étale topology, and let $\operatorname{SH}\left(\operatorname{Shv}\left((\operatorname{Spec} F)_{\text {rét }}\right)\right)$ denote the local stable homotopy category of sheaves of spectra on $(\operatorname{Spec} F)_{\text {rét }}$ (see [3, Section 2]). Let $X_{F}$ denote the Harrison space of orderings on $F$. By [27, Theorem 1.3], $\operatorname{Shv}\left(X_{F}\right) \simeq$ $\operatorname{Shv}\left((\operatorname{Spec} F)_{\text {rét }}\right)$. Bachmann's theorem (specialized to the case in which the base scheme is Spec of a field) then tells us the following.

THEOREM 4.5 [3, Theorem 31]. There are triangulated equivalences of categories

$$
\operatorname{SH}^{\mathbb{A}^{1}}(F)[1 / \rho] \simeq \operatorname{SH}\left(\operatorname{Shv}\left((\operatorname{Spec} F)_{\text {rét }}\right)\right) \simeq \operatorname{SH}\left(\operatorname{Shv}\left(X_{F}\right)\right) .
$$


Because of the relation $(2+\rho \eta) \eta=0$, we see that $\rho$ is invertible whenever 2 and $\eta$ are invertible. From this, Bachmann derives the following corollary.

COROLLARY 4.6. There are triangulated equivalences of categories

$$
\mathrm{SH}^{\mathbb{A}^{1}}(F)[1 / 2,1 / \eta] \simeq \operatorname{SH}\left(\operatorname{Shv}\left((\operatorname{Spec} F)_{\text {rét }}\right)\right)[1 / 2] \simeq \operatorname{SH}\left(\operatorname{Shv}\left(X_{F}\right)\right)[1 / 2]
$$

REMARK 4.7. Note that when $X_{F}=*$, we have $\operatorname{SH}\left(\operatorname{Shv}\left(X_{F}\right)\right)=\mathrm{SH}$, the classical Spanier-Whitehead category. When $X_{F}=*$ and $F$ admits a real embedding, the equivalences in Theorem 4.5 and Corollary 4.6 come from the real Betti realization functor [3, Corollary 38].

4.3. Uncompletion. We now use Bachmann's theorem and several fracture squares to prove Theorem 1.6.

Proof of Theorem 1.6. We prove the vanishing statement for $\underline{\pi}_{\star} \mathbb{1}$; the reader may check that an analogous argument easily covers the $p$-local version.

By Lemma 4.4, it suffices to show that $\underline{\pi}_{\star} \mathbb{1}_{k}$ obtains the stated vanishing range for all $k$ formally real with $\operatorname{vcd}_{2} k<\infty$ or nonreal of characteristic 0 . The vanishing range in Theorem 1.6 is a subset of the range from Theorem 1.4, so the latter case is covered. Now suppose $k$ is formally real with $\operatorname{vcd}_{2} k<\infty$. We claim that it suffices to check that the homotopy groups $\pi_{\star} \mathbb{1}_{k}$ obtain the vanishing range. Indeed, if $E / k$ is a finitely generated field extension, Lemma 4.1 implies that $\underline{\pi}_{m+n \alpha} \mathbb{1}_{k}(\operatorname{Spec} E)=\pi_{m+n \alpha} \mathbb{1}_{E}$. We either have that $E$ is nonreal of characteristic 0 (and can invoke Theorem 1.4), or that $E$ is formally real. In the latter case, the results of [28, Section II.4.1 \& II.4.2] imply that $\operatorname{vcd}_{2} E<\infty$, and we are still working with a homotopy group over a field satisfying our hypotheses. Thus we have successfully reduced the problem to checking the vanishing range of $\pi_{\star} \mathbb{1}_{k}$ for $k$ formally real with $\operatorname{vcd}_{2} k<\infty$.

Fix $F$ formally real with finite $\mathrm{vcd}_{2}$ and consider the following three homotopy pullback squares:
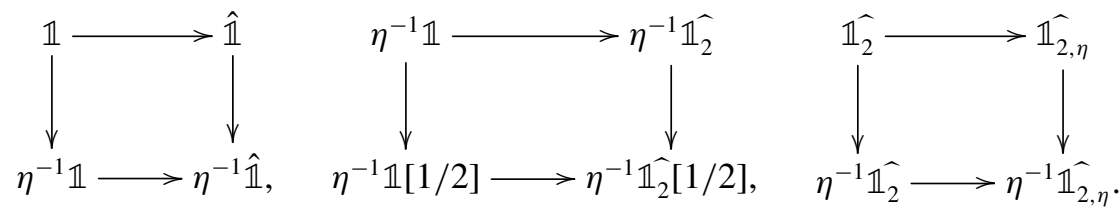

The first is the $\eta$-primary fracture square for $\mathbb{1}$, the second is the $\eta$-periodization of the 2-primary fracture square for $\mathbb{1}$, and the third is the $\eta$-primary fracture square 
for $\widehat{\mathbb{1}_{2}}$. The vanishing ranges for $\pi_{\star} \hat{\mathbb{1}}$ and $\pi_{\star} \eta^{-1} \hat{\mathbb{1}}$ follow from Theorem 1.1 , so the first square implies that it suffices to check the vanishing range for $\pi_{\star} \eta^{-1} \mathbb{1}$.

This brings us to the second square. We analyze the bottom row using Corollary 4.6, which tells us that $\pi_{m+n \alpha} \eta^{-1} \mathbb{1}[1 / 2]=0$ if and only if $\mathbb{1}[1 / 2] \in$ $\operatorname{SH}\left(\operatorname{Shv}\left(X_{F}\right)\right)$ has 0 as its $m$ th homotopy group. We claim that this latter condition is obtained if and only if $\pi_{m}^{\text {top }} \mathbb{1}[1 / 2]=0$. By the argument of [3, Proposition 40], it suffices to check this condition when $F$ is real closed. But then $X_{F}=*$ and $\mathrm{SH}\left(\operatorname{Shv}\left(X_{F}\right)\right)=\mathrm{SH}$, which is precisely the category in which $\pi_{m}^{\text {top }}$ is computed. By the same argument, $\pi_{m+n \alpha} \eta^{-1} \hat{\mathbb{1}_{2}}[1 / 2]=0$ if and only if $\pi_{m}^{\text {top }} \hat{\mathbb{1}_{2}}[1 / 2]=0$. (Note that by Serre finiteness, the set of such $m$ is a subset of those for which $\pi_{m}^{\text {top }} \mathbb{1}[1 / 2]=0$.)

It remains to check the vanishing range for $\pi_{\star} \eta^{-1} \widehat{\mathbb{1}_{2}}$. Since $\operatorname{ved}_{2}(F)<\infty,[13$, Theorem 1] implies that the top row of the third square is a $\pi_{\star}$-isomorphism, whence the bottom row is a $\pi_{\star}$-isomorphism as well. In particular, $\pi_{m+n \alpha} \eta^{-1} \hat{\mathbb{1}_{2}}=$ 0 if and only if $\pi_{m+n \alpha} \eta^{-1} \widehat{\mathbb{1}_{2, \eta}}=0$. By Theorem 1.1, this condition holds whenever $m<0$ or $m>0, m \equiv 1$ or $2(\bmod 4)$, and $2 n>\max \{3 m+5,4 m\}$. It follows that $\pi_{m+n \alpha} \eta^{-1} \mathbb{1}=0$ whenever $m<0$ or $m>0, m \equiv 1$ or $2(\bmod 4), 2 n>$ $\max \{3 m+5,4 m\}$, and $\pi_{m}^{\text {top }} \mathbb{1}[1 / 2]=0$. This concludes our proof.

\section{Questions}

Here we present several natural questions raised by our work, along with some commentary.

Question 5.1. Given $m \in \mathbb{Z}$ such that the $m$ th $\eta$-complete Milnor-Witt stem $\underline{\pi}_{m+* \alpha} \hat{\mathbb{1}}$ is bounded above, what is the smallest $n \in \mathbb{Z}$ such that $\underline{\pi}_{m+n \alpha} \hat{\mathbb{1}}=0$ ? If the $m$ th Milnor-Witt stem $\underline{\pi}_{m+* \alpha} \mathbb{1}$ is bounded above, what is the smallest $n$ such that $\underline{\pi}_{m+n \alpha} \mathbb{1}=0$ ?

REMARK 5.2. The bounds presented here are not necessarily optimal. For instance, by [26], $\underline{\pi}_{1+3 \alpha} \mathbb{1}=0$, but the vanishing region of Theorem 1.6 is only obtained for $\underline{\pi}_{1+n \alpha} \mathbb{1}$ when $n>4$. From the perspective of the slice spectral sequence, we lack both total information about the Novikov $E_{2}$-page and all the differentials in the spectral sequence. While improvements on the vanishing range are no doubt possible via more nuanced slice arguments, it seems likely that different arguments would have to be invoked in order to find optimal bounds.

Suppose that the $m$ th Milnor-Witt sheaf is bounded above and take $n$ to be the maximal integer such that $\underline{\pi}_{m+n \alpha} \mathbb{1} \neq 0$. In this case, we will call $\underline{\pi}_{m+n \alpha} \mathbb{1}$ the top sheaf in the $m$ th Milnor-Witt stem. 
Recall that $\omega \mathscr{F}$ denotes the contraction of a sheaf $\mathscr{F}$ [20]. Tom Bachmann pointed out to the authors that $\omega \mathscr{F}=0$ if and only if $\mathscr{F}$ is birational, that is, if and only if every dense open embedding of smooth $F$-schemes $U \rightarrow X$ induces an isomorphism $\mathscr{F}(X) \cong \mathscr{F}(U)$. One implication is straightforward; the other can be deduced from the Rost-Schmid complex. Together with the fact that $\omega \underline{\pi}_{m+n \alpha} \cong$ $\underline{\pi}_{m+(n+1) \alpha}$, this implies that every top Milnor-Witt sheaf is birational.

QUESTION 5.3. Are there examples of top Milnor-Witt sheaves that are birational but not constant?

REMARK 5.4. Matthias Wendt informed the authors about [2, Lemma 3.7], which implies that $\omega \underline{K}_{3}^{\text {ind }}=0$, where $\underline{K}_{3}^{\text {ind }}$ is the third indecomposable $K$-sheaf. Note that $\underline{K}_{3}^{\text {ind }}$ coincides with the sheaf of integral motivic cohomology groups $\underline{H}^{1,2}$, which is nonzero and nonconstant. For example, $H^{1,2}(\mathbb{Q}) \cong \mathbb{Z} / 24$ and $H^{1,2}(\mathbb{Q}(\sqrt{-1}))$ contains $\mathbb{Z}$ as a direct summand; see, for example, [16, pp. 542, 564].

There is no indication that $\underline{K}_{3}^{\text {ind }}$ appears as a top Milnor-Witt sheaf. All known top Milnor-Witt sheaves are constant.

We conclude by noting that the methods of Section 3 imply another result, whose proof we only sketch.

THEOREM 5.5. The natural map $\mathbb{1} \rightarrow \eta^{-1} \mathbb{1}$ induces an isomorphism $\underline{\pi}_{m+n \alpha} \mathbb{1} \cong$ $\underline{\pi}_{m+n \alpha} \eta^{-1} \mathbb{1}$ whenever

$\gg m<0$, or

$» m \geqslant 0$ and $2 n>\max \{3 m+5,4 m\}$.

Proof Sketch. By the $\eta$-primary fracture square, it suffices to prove the analogous result for $\hat{\mathbb{1}} \rightarrow \eta^{-1} \hat{\mathbb{1}}$.

We compare the weight $n$ slice spectral sequence for $\mathbb{1}$ to the weight $n$ $\eta$-inverted slice spectral sequence. We get an isomorphism on $E_{1}$-pages above the slice-to-Novikov shift of the Andrews-Miller region from Lemma 2.6. This is precisely the region stated in the theorem.

REMARK 5.6. By Morel's computation of $\underline{\pi}_{n \alpha} \mathbb{1}$, the isomorphism in fact holds for $n>0$ when $m=0$.

REMARK 5.7. By Theorem 5.5 and the fracture squares from the proof of Theorem 1.6, we can also deduce a relative version Theorem 1.6. Assume that $m$, 
$n$ are in the range given in Theorem 5.5 so that $\underline{\pi}_{m+n \alpha} \mathbb{1} \cong \underline{\pi}_{m+n \alpha} \eta^{-1} \mathbb{1}$. Then the $\eta$-periodization of the 2-primary fracture square produces a long exact sequence

$$
\begin{aligned}
\cdots & \rightarrow \underline{\pi}_{m+1+n \alpha} \eta^{-1} \widehat{\mathbb{1}_{2}}[1 / 2] \rightarrow \underline{\pi}_{m+n \alpha} \eta^{-1} \mathbb{1} \\
& \rightarrow \underline{\pi}_{m+n \alpha} \eta^{-1} \mathbb{1}[1 / 2] \oplus \underline{\pi}_{m+n \alpha} \eta^{-1} \underline{\mathbb{1}}_{2} \rightarrow \cdots .
\end{aligned}
$$

The terms $\underline{\pi}_{m+1+n \alpha} \eta^{-1} \mathbb{1}$ and $\underline{\pi}_{m+n \alpha} \eta^{-1} \mathbb{1}[1 / 2]$ are topological in the sense that they can be computed in $\operatorname{SH}\left(\operatorname{Shv}\left(X_{F}\right)\right)$ using Corollary 4.6. Since we are in the range of Theorem 5.5, the argument from the final paragraph of the proof of Theorem 1.6 implies that $\pi_{m+n \alpha} \eta^{-1} \hat{\mathbb{1}}_{2}=0$. This finally gives us a range in which $\pi_{m+n \alpha} \mathbb{1}$ is 'topological', at least in the sense of coming from $\operatorname{SH}\left(\operatorname{Shv}\left(X_{F}\right)\right)$ via the above long exact sequence.

Question 5.8. For $m>0,2 n>\max \{3 m+5,4 m\}$, and $n \equiv 0$ or $3(\bmod 4)$, what is $\underline{\pi}_{m+n \alpha} \mathbb{1}$ ? What about $\underline{\pi}_{m+n \alpha} \hat{\mathbb{1}}$ ?

REMARK 5.9. By Theorem 5.5, this is equivalent to computing the homotopy sheaves of the $\eta$-inverted sphere spectrum. The global sections of these sheaves are computed for $F=\mathbb{C}$ in [1], and, for $F=\mathbb{R}$, the 2-complete global section computation appears in [8]. Calculations for $p$-adic fields $\mathbb{Q}_{p}$ and the rational numbers $\mathbb{Q}$ will appear in [36]. Any sheaf computations and computations over a general field are completely open, except for $\underline{\pi}_{1+m \alpha} \eta^{-1} \mathbb{1}=\underline{\pi}_{2+m \alpha} \eta^{-1} \mathbb{1}=0$ by [25, Theorem 8.3], which predates the vanishing known to occur under the conditions of Theorem 1.6.

\section{Acknowledgements}

The authors thank the anonymous referee for a number of helpful suggestions that improved the exposition. The authors gratefully acknowledge hospitality and support by the Mathematisches Forschungsinstitut Oberwolfach during Summer 2016 and the Institut Mittag-Leffler during Spring 2017. The first author was supported by NSF awards DMS-1406327 and DMS-1709302, the second author was supported by the DFG priority programme 'Homotopy theory and algebraic geometry', and the third author was supported by the RCN Frontier Research Group Project (no. 250399) 'Motivic Hopf equations' and a Friedrich Wilhelm Bessel Research Award from the Humboldt Foundation.

\section{References}

[1] M. Andrews and H. Miller, 'Inverting the Hopf map', J. Topol. 10(4) (2017), 1145-1168.

[2] A. Asok and J. Fasel, 'Algebraic vector bundles on spheres', J. Topol. 7(3) (2014), 894-926. 
[3] T. Bachmann, 'Motivic and real etale stable homotopy theory', Preprint, 2017, arXiv:1608.0 $8855 \mathrm{v} 2$.

[4] D. Dugger and D. C. Isaksen, 'The motivic Adams spectral sequence', Geom. Topol. 14(2) (2010), 967-1014.

[5] D. Dugger and D. C. Isaksen, ' $\mathbb{Z} / 2$-equivariant and $\mathbb{R}$-motivic stable stems', Preprint, 2016, arXiv:1603.09305.

[6] D. Dugger and D. C. Isaksen, 'Low-dimensional Milnor-Witt stems over $\mathbb{R}$ ', Ann. K-Theory 2(2) (2017), 175-210.

[7] B. Gheorghe and D. C. Isaksen, 'The structure of motivic homotopy groups', Bol. Soc. Mat. Mexicana (3) 23(1) (2017), 389-397.

[8] B. J. Guillou and D. C. Isaksen, 'The $\eta$-inverted $\mathbb{R}$-motivic sphere', Algebr. Geom. Topol. 16(5) (2016), 3005-3027.

[9] J. Heller and K. Ormsby, 'Galois equivariance and stable motivic homotopy theory', Trans. Amer. Math. Soc. 368(11) (2016), 8047-8077.

[10] J. Heller and K. Ormsby, 'The stable Galois correspondence for real closed fields', Preprint, 2017, arXiv:1701.09099.

[11] J. Hornbostel and S. Yagunov, 'Rigidity for henselian local rings and $\mathbb{A}^{1}$-representable theories', Math. Z. 255(2) (2007), 437-449.

[12] M. Hoyois, 'From algebraic cobordism to motivic cohomology', J. Reine Angew. Math. 702 (2015), 173-226.

[13] P. Hu, I. Kriz and K. Ormsby, 'Convergence of the motivic Adams spectral sequence', J. K-Theory 7(3) (2011), 573-596.

[14] P. Hu, I. Kriz and K. Ormsby, 'Remarks on motivic homotopy theory over algebraically closed fields', J. K-Theory 7(1) (2011), 55-89.

[15] M. Levine, 'Convergence of Voevodsky's slice tower', Doc. Math. 18 (2013), 907-941.

[16] A. S. Merkurjev and A. A. Suslin, 'The group $K_{3}$ for a field', Izv. Akad. Nauk SSSR Ser. Mat. 54(3) (1990), 522-545.

[17] F. Morel, 'Suite spectrale d'Adams et invariants cohomologiques des formes quadratiques', C. R. Acad. Sci. Paris Sér. I Math. 328(11) (1999), 963-968.

[18] F. Morel, 'On the motivic $\pi_{0}$ of the sphere spectrum', in Axiomatic, Enriched and Motivic Homotopy Theory, NATO Sci. Ser. II Math. Phys. Chem., 131 (Kluwer Academic Publishers, Dordrecht, 2004), 219-260.

[19] F. Morel, 'The stable $\mathbb{A}^{1}$-connectivity theorems', K-Theory 35(1-2) (2005), 1-68.

[20] F. Morel, $\mathbb{A}^{1}$-Algebraic Topology over a Field, Lecture Notes in Mathematics, 2052 (Springer, Heidelberg, 2012).

[21] S. P. Novikov, 'Methods of algebraic topology from the point of view of cobordism theory', Izv. Akad. Nauk SSSR Ser. Mat. 31 (1967), 855-951.

[22] K. Ormsby, Computations in stable motivic homotopy theory. ProQuest LLC, Ann Arbor, MI, PhD Thesis, University of Michigan, 2010.

[23] K. M. Ormsby and P. A. Østvær, 'Stable motivic $\pi_{1}$ of low-dimensional fields', Adv. Math. 265 (2014), 97-131.

[24] D. C. Ravenel, Complex Cobordism and Stable Homotopy Groups of Spheres, Pure and Applied Mathematics, 121 (Academic Press, Inc., 1986).

[25] O. Röndigs, 'On the $\eta$-inverted sphere'. Proceedings of the International Colloquium on $K$ Theory, TIFR, to appear, Preprint, 2016, arXiv:1602.08798.

[26] O. Röndigs, M. Spitzweck and P. A. Østvær, 'The first stable homotopy groups of motivic spheres', Preprint, 2016, arXiv:1604.00365. 
[27] C. Scheiderer, Real and Étale Cohomology, Lecture Notes in Mathematics, 1588 (Springer, Berlin, 1994).

[28] J.-P. Serre, Galois Cohomology, Springer Monographs in Mathematics (Springer, Berlin, 2002).

[29] A. Suslin and V. Voevodsky, 'Bloch-Kato conjecture and motivic cohomology with finite coefficients', in The Arithmetic and Geometry of Algebraic Cycles (Banff, AB, 1998), NATO Sci. Ser. C Math. Phys. Sci., 548 (Kluwer Academic Publishers, Dordrecht, 2000), 117-189.

[30] A. Suslin and V. Voevodsky, 'Singular homology of abstract algebraic varieties', Invent. Math. 123(1) (1996), 61-94.

[31] H. Toda, Composition Methods in Homotopy Groups of Spheres, Annals of Mathematics Studies, 49 (Princeton University Press, Princeton, N.J., 1962).

[32] V. Voevodsky, ' $\mathbf{A}^{1}$-homotopy theory', in Proceedings of the International Congress of Mathematicians, Vol. I (Berlin, 1998), Extra Vol. I (1998), 579-604.

[33] V. Voevodsky, 'Open problems in the motivic stable homotopy theory. I', in Motives, Polylogarithms and Hodge Theory, Part I (Irvine, CA, 1998), Int. Press Lect. Ser., 3 (International Press, Somerville, MA, 2002), 3-34.

[34] V. Voevodsky, 'Motivic cohomology with Z/2-coefficients', Publ. Math. Inst. Hautes Études Sci. 98 (2003), 59-104.

[35] V. Voevodsky, 'On motivic cohomology with Z/l-coefficients', Ann. of Math. (2) 174(1) (2011), 401-438.

[36] G. M. Wilson, 'The $\eta$-inverted motivic sphere over the rationals', Algebr. Geom. Topol. In preparation, arXiv:1708.06523.

[37] G. M. Wilson and P. A. Østvær, 'Two-complete stable motivic stems over finite fields', Algebr. Geom. Topol. 17(2) (2017), 1059-1104.

[38] R. Zahler, 'The Adams-Novikov spectral sequence for the spheres', Ann. of Math. (2) 96 (1972), 480-504. 